Again, if the results of the two tests are independent, the likelihood of two false-negative results drops to $9 \%$.

The implication is clear: even an inaccurate test tells us something. Or, to misquote the World Health Organization: 'test, re-test, re-test'.

Use of this strategy would be made easier if there were a database - updated in real time - of point-of-care tests being generated by labs around the world. This database, which could be assembled by an international organization such as the World Health Organization, would list the lab and test name, the antibody that the test detects (e.g., IgG, IgN or both ${ }^{7}$ ), the detection method (e.g., lateral-flow immunoassay) and its accuracy and reproducibility, the turnaround time, the testing-kit cost and the sample-processing cost. With this information in hand, governments and international organizations could advise scientists on what combination of cheap tests would be optimal for specific nations.

Third, consider a quick and cheap test with a $30 \%$ false-positive rate, and for simplicity, zero false-negative results. First, one could test many people with this test, and then test the subset who test positive with a highly accurate test. This economizes on the use of scarce but accurate test kits while allowing much wider testing than would have been possible with the few accurate test kits available. In short: 'test, triage, re-test'.

Finally, smart tactics can enable cheaper testing with the expensive RT-PCR tests, if a sample obtained can 'fuel' multiple tests. Some German hospitals are doing 'block tests' using a sample pooled from ten employees, and then are testing individually only if there is a positive result ${ }^{8}$.

One can take this idea further, by applying principles from discrete optimization. If the test is positive, then one would test two blocks of five samples each, and then further test the arm that tests positive. This mimics 'branch and bound' algorithms for solving discrete optimization problems such as the famous 'traveling salesperson' problem ${ }^{9}$, which requires finding the cheapest route for delivering supplies to a fixed number of stores.

These simple examples are illustrative. Naturally, several factors would come into play in their implementation. For example, block testing would increase time to diagnosis and may be more useful for asymptomatic low-risk cases.

Finally, all inaccuracies are not equal. Right now, tests with a high false-positive rate are less problematic - since people are being advised to stay home anyway - than those with high false-negative rates. Furthermore, a false-positive result for SARS-CoV-2 is unlikely to initiate treatment with negative side effects, as would chemotherapy for misdiagnosed cancer.

The key point here is that creative use of currently available cheap and quick tests - even if they are inaccurate and unreproducible - can go a long way to reaching adequate levels of accuracy and precision, at least until the gold-standard tests can be developed. of Surgery \& Cancer, Imperial College London, London, UK. ${ }^{3}$ Department of Economics, University of Oxford, Oxford, UK.

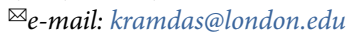

Published online: 12 May 2020

https://doi.org/10.1038/s41591-020-0891-7

\section{References}

1. Boseley, S. \& Kollewe, J. The Guardian https://www.theguardian. com/world/2020/mar/26/covid-19-self-test-could-allow-returnto-work-says-public-health-england (26 March 2020).

2. Boseley, S. The Guardian https://www.theguardian.com/world/ 2020/mar/25/uk-coronavirus-mass-home-testing-to-be-madeavailable-within-days (25 March 2020).

3. Ghebreyesus, T. A. \& Swaminathan, S. Lancet $\mathbf{3 9 5}$, 762-764 (2020).

4. Mahmud, F. Al Jazeera https://www.aljazeera.com/news/2020/ 03/bangladesh-scientists-create-3-kit-detect-covid-19200323035631025.html (24 March 2020)

5. Devlin, H. The Guardian https://www.theguardian.com/world/ 2020/apr/05/coronavirus-testing-kits-could-be-unreliableuk-scientists-say (5 April 2020).

6. Centers for Disease Control and Prevention. https://www.cdc. gov/flu/professionals/diagnosis/rapidlab.htm (4 February 2019).

7. Center for Health Security, Johns Hopkins School of Public Health. http://www.centerforhealthsecurity.org/resources/ COVID-19/200228-Serology-testing-COVID.pdf (28 February 2019).

8. Bennhold, K. The New York Times https://www.nytimes.com/ 2020/04/04/world/europe/germany-coronavirus-death-rate.html (4 April 2020).

9. Fisher, M. L. Manage. Sci. 27, 1-18 (1981).

\section{Acknowledgements}

We thank London Business School for providing Research and Materials Development funding in support of this research. Infrastructure support for this research was provided by the National Institute of Health Research Imperial Biomedical Research Centre.

\section{Competing interests}

A.D. has received a donation of one Axceed 260 machine from Bioscience Diagnostic Medical Technology in China, and is the academic sponsor on a study focusing on PCR and antibody testing for COVID-19 on behalf of the Department of Health.

\title{
The fight to end tuberculosis must not be forgotten in the COVID-19 outbreak
}

To the Editor - In the midst of the COVID19 pandemic, the world must remain vigilant to the potential for eruptions of tuberculosis (TB) and its drug-resistant (DR-TB) strains. Many countries with a high TB burden remain reliant on in-person and community-based directly observed therapy for TB treatment. With the current COVID19 conditions that affect mobility and access to care ${ }^{1-4}$, bothw seem impractical.

Global TB is exacerbated by the COVID19 pandemic. Regular treatment facilities are being closed because of a lack of resources, and TB could be misdiagnosed in settings in which COVID-19 testing is not available. Local governments must identify feasible options to retain patients with TB and DR-TB in care while fighting the COVID-19 pandemic. On 20 March 2020, the World Health Organization released an Information Note on TB and COVID-19 urging national TB programs to maintain continuity of essential services for people affected with TB during the COVID-19 pandemic ${ }^{5}$. It recommends providing adequate stocks of
TB medicines for all patients in order to ensure treatment completion without the patients' having to visit treatment centers unnecessarily to collect medications; this essentially forces the global TB program to shift from directly observed therapy to self-administered therapy. Digital-health technologies such as electronic medication monitors and video-supported therapy were also recommended to help patients adhere to their treatment. However, for optimal implementation of this strategy, trials evaluating the effectiveness of remote 
treatment for TB in low- and middle-income countries are desperately needed. Healthcare workers urgently need to disseminate information on how to address patients with TB and DR-TB in the current COVID-19 outbreak. The most common medications used to treat TB and DR-TB need to be adequately stocked, primarily in countries with a high burden, to sustain clinical services in case of further restrictions due to COVID-19. For countries in sub-Saharan Africa where the healthcare system is fragile in withstanding the COVID-19 outbreak ${ }^{6-9}$, responding to these two diseases at the same time needs due diligence.

There have been several efforts contributing to the global TB momentum, including the End-TB Strategy, and tremendous efforts have been made to halt the TB epidemic. Undoing those gains would undoubtedly increase global health-security tension and result in deadly economic, social, political and health consequences. Both COVID-19 and TB have no borders, and both require a major commitment from all key stakeholders.

\section{Tsegahun Manyazewal(iD) 1⿴, Yimtubezinash Woldeamanuel', Henry M. Blumberg'ㄹ, Abebaw Fekadu' and Vincent C. Marconi \\ ${ }^{1}$ Addis Ababa University, College of Health Sciences, Center for Innovative Drug Development and Therapeutic Trials for Africa, Addis Ababa, Ethiopia. ${ }^{2}$ Emory University School of Medicine and Rollins School of Public Health, Atlanta, GA, USA. \\ 凶e-mail: tsegahunm@gmail.com}

Published online: 12 May 2020 https://doi.org/10.1038/s41591-020-0917-1

\section{References}

1. Arnold, C. Nat. Med. https://doi.org/10.1038/d41591-020-00005-1 (2020).

2. Gibney, E. Nature 580, 176-177 (2020).
Bhadelia, N. Nature 578, $193(2020)$

4. Anderson, R. M., Heesterbeek, H., Klinkenberg, D. \& Hollingsworth, T. D. Lancet 395, 931-934 (2020).

5. World Health Organization. https://www.who.int/tb/ COVID_19considerations_tuberculosis_services.pdf (accessed 23 March 2020).

6. Maxmen, A. Nature 580, 173-174 (2020)

7. Nkengasong, J. N. \& Mankoula, W. Lancet 395, 841-842 (2020).

8. Adepoju, P. Nat. Med. 26, 444-448 (2020).

9. Makoni, M. Lancet 395, 483 (2020).

Acknowledgements

Supported by the Fogarty International Center and National Institute of Allergy and Infectious Diseases of the US National Institutes of Health under Award Number D43TW009127. The content is solely the responsibility of the authors and does not necessarily represent the official views of the National Institutes of Health.

Author contributions

T.M. conceived of the idea presented and developed the first draft; V.C.M., Y.W., H.M.B. and A.F. revised the draft; and all authors approved the final version for publication.

Competing interests

The authors declare no competing interests. 